\title{
A proteomic approach to understand the impact of nodulation on salinity stress response in alfalfa (Medicago sativa L.)
}

\section{Yafang Wang}

Northwest A\&F University

\section{Pan Zhang}

Northwest A\&F University

Tianming $\mathrm{Hu}$

Northwest A\&F University

Yajun Wu

South Dakota State University

Peizhi Yang ( $\nabla$ yangpeizhi@126.com )

Northwest Agriculture and Forestry University https://orcid.org/0000-0002-5937-837X

Research article

Keywords: iTRAQ; oxidative stress; plant-microbe interaction; root nodules; symbiotic nitrogen fixation

Posted Date: September 9th, 2019

DOI: https://doi.org/10.21203/rs.2.14197/v1

License: (c) (i) This work is licensed under a Creative Commons Attribution 4.0 International License. Read Full License

Version of Record: A version of this preprint was published at Plant Biology on December 6th, 2021. See the published version at https://doi.org/10.1111/plb.13369. 


\section{Abstract}

Background Symbiotic nitrogen fixation in legumes is an important source of nitrogen supply in sustainable agriculture. Salinity is a key abiotic stress that negatively affects host plant growth, rhizobium-legume symbiosis and nitrogen fixation.Results To explore how the symbiotic relation impacts plant response to salinity, we assayed the proteome profile of alfalfa plants with active nodules (NA), inactive nodules $(\mathrm{NI})$ or without nodules $(\mathrm{NN})$ when plants were subjected to salinity stress. Our data suggested that NA plants respond to salinity stress through some unique signaling regulations. NA plants showed an upregulation of proteins related to cell wall remodeling and reactive oxygen species (ROS) scavenging and a down-regulation of proteins involved in protein synthesis and degradation. The data also showed that NA plants, together with NI plants, upregulated proteins in photosynthesis, carbon fixation and respiration, anion transport, and plant defense to pathogens.Conclusions The data suggest that the symbiotic relations conferred the host plant a better capacity to adjust the key processes, probably to more efficiently use energy and resources, deal with oxidative stress, and maintain ion homeostasis and healthy status during salinity stress.

\section{Background}

Symbiotic nitrogen fixation (SNF), a key point for the entry of atmospheric nitrogen into the terrestrial ecosystem, is the most important biologically mediated process after photosynthesis [1]. It is an important source of nitrogen supply in sustainable agriculture and helps in limiting the use of nitrogen fertilizer at the economic and agricultural level [2]. Because of these reasons, many legumes that can establish a nitrogen-fixing symbiosis with soil bacteria, generally known as rhizobia, are among the most important agricultural crops [3].

In the rhizobium-legume symbiotic process, host plants provide carbon compounds derived from photosynthesis to support rhizobia activity and the development of microbial populations. In return, rhizobial symbiosis improves plants' nutritional status by providing fixed nitrogen, promotes plant development, and enhances their defense against pathogens and pests and their adaptation to various environmental stresses [4]. Rhizobia produce chemical molecules that can influence plant development, including phytohormones, lipo-chito-oligosaccharide nod factors, lumichrome, riboflavin, and $\mathrm{H}_{2}$ evolved by nitrogenase [5]. When present in soil, nod factors can stimulate seed germination, promote plant growth, and increase grain yields, as well as increase photosynthetic rates. Very low concentrations of lumichrome and $\mathrm{H}_{2}$ released by rhizobia also promote plant growth and increase biomass. Rhizobia are known to suppress the population of soil pathogens, and the legume itself releases phenolics that can suppress pathogens and promote growth of mutualistic microbes. Rhizobial inoculation of plants has also been suggested to alleviate the effect of water stress in symbiotic legumes [6, 7]. It is possible that rhizobial products such as cytokinin, which results in ABA accumulation, elicits stomata closure and thus decreases water loss via transpiration in the leaves $[5,8]$. It has been reported that a response observed 
during one type of stress may have a role in the amelioration of another stress [9]. The symbiosis thus may enhance the fitness of their hosts and improve their tolerance to abiotic stresses.

Salinity, one of the most important abiotic stresses, results in low agricultural productivity, low economic returns, and soil erosions $[10,11]$. Legumes growing in saline environments exhibit reduced yield [12]. It was reported that rhizobium-legume symbiosis and nitrogen fixation in root nodules are more sensitive to salinity than plant growth [13]. The higher sensitivity of rhizobium-legume symbiosis and nitrogen fixation may be due to two main possibilities: rhizobium is more sensitive to salinity compared to host plants, or plants sever the symbiotic relation to conserve energy in stress response. The latter leads to other frequently asked questions: i.e. how the symbiotic relation impacts plant tolerance to salinity. It has been reported that $\mathrm{N}$-fixing legumes were more sensitive to salt stress than those depending on mineral nitrogen [14]. Compared with $\mathrm{N}$-fertilized plants, the ones with SNF showed a larger degree of decreased yield [15], less nitrogen accumulation [16] and greater uptake of $\mathrm{Na}^{+}$and $\mathrm{Cl}^{-}$under $\mathrm{NaCl}$ stress, inducing ionic imbalance in host plants [17]. Interestingly, Cordovilla et al.[18] reported that peas (Pisum sativum L.) dependent on nitrogen fixation were more tolerant to salt stress than those $\mathrm{N}$-fertilized. In another study, chickpea (Cicer arietinum) genotypes with a greater capacity for nodulation performed better than other genotypes under salt stress in terms of both $\mathrm{N}$ fixation and grain yield [19]. Opposite results were obtained with faba bean (Vicia faba L.), where growth inhibition of shoot and root was observed in nitrogen-fixing plants [18]. Apparently, more studies are needed to address how the symbiotic relationship impacts salt tolerance in legume plants and to understand the physiological and molecular processes of stress response that are affected by SNF.

Salt tolerance is a complex phenomenon that involves morphological and developmental changes as well as physiological and biochemical processes. Many salt tolerant plants adapt to salinity by minimizing direct effects of $\mathrm{Na}$ or $\mathrm{Cl}$ ions through mechanisms such as salt exclusion, salt sequestration or salt excretion [11]. However, these mechanisms are usually not sufficient in limiting the amount of salt entering the plants. Thus, many plants need to deal with the direct effects of salt toxicity. One adverse effect of salinity is production of reactive oxygen species (ROS) [20]. ROS not only damage host plants but also negatively affect nitrogen fixation [21]. Prevention of ROS production in the nodule and reduction of oxidative stress could provide a protective effect on nodule structure and function in salinity stress [22]. Plants employ antioxidants (e.g., ascorbate and glutathione) and detoxifying enzymes, such as superoxide dismutase (SOD), catalase (CAT), and ascorbate peroxidase (APX), to combat oxidative stress induced by salinity $[23,24]$. Dehydration often occurs concurrently with salinity stress, and accumulation of solutes, like proline and soluble sugars, helps plant systems adapt to a saline environment by reducing water loss [25]. Plants accumulate proline, soluble sugar and stress responsive proteins that participate actively in the osmotic adjustment when plants are under salt stress [26, 27]. 
A number of approaches have been used to identify genes and proteins that are involved in plant salt tolerance, such as screening cDNA libraries to isolate salt-responsive cDNA clones and analyzing gene expression at the transcriptional and post-transcriptional levels using transcript profiling [28]. A number of genes involved in salt tolerance and symbiotic nitrogen fixation have been isolated and characterized [29]. Proteomics is a powerful tool for describing the complete proteome and for comparing how the proteome is affected by different internal and environmental factors. Proteomic responses of rice [30], grasspea [31], sorghum [32], common wheat [33], soybean [34, 35], and many other plants to salt stress have been reported. Theses proteomics experiments detected many proteins involved in plant response to salt stress. For example, an increased accumulation of enzymes involved in glycolysis and carbohydrate metabolism is frequently observed, indicating a greater need for energy during stress response. Other major groups of increased proteins are ROS scavenging enzymes, suggesting dealing with oxidative stress induced by salinity is one of the important processes in stress response. Other proteins with an increased abundance under salinity are enzymes involved in biosynthesis of compatible solutes [36]. However, how these stress responsive proteomes are affected by SNF has not been studied.

Alfalfa (Medicago sativa L.) is the most extensively cultivated leguminous forage in the world. Although alfalfa is considered a moderately salt tolerant species when compared to other legumes, salinity is one of the limiting factors in the production and nitrogen-fixing activity of alfalfa [37,38]. While salinity inhibits both plant production and rhizobium-mediated nitrogen-fixing activity in alfalfa, it is unclear how the symbiotic interaction affects salinity response in plants. We recently demonstrated that symbiosis in alfalfa improves plant survival to salinity treatment [39]. We hypothesize that symbiotic relations result in molecular and physiological changes that contribute to greater salt tolerance in plants. To address this hypothesis, we studied proteomic changes in alfalfa plants under salinity stress with or without symbiotic relation with rhizobium. Specifically, the isobaric tags for relative and absolute quantification (iTRAQ)based proteomic approaches were used to quantify protein changes in alfalfa plants with active nodules, inactive nodules, or no nodules when plants were subjected to salinity stress. We also examined relative water content, antioxidant enzymatic activities, and osmotic solute content as a few key physiological changes in these plants.

\section{Methods}

\section{Plant materials and growth conditions}

Alfalfa seeds (Medicago sativa L. cv. Ladak ${ }^{+}$) and rhizobia (Rhizobium meliloti strain Dormal) used in this study were purchased from the Clover Group of China. Seeds were surface sterilized with $70 \%$ ethanol for $30 \mathrm{~s}$ and $5 \%$ sodium hypochlorite for 5 min, rinsed 4 to 5 times with sterile water, and 
germinated on wet filter paper in Petri dishes in a growth chamber at $25 / 15^{\circ} \mathrm{C}, 55 / 70 \%$ relative humidity (day/night). Five-day-old seedlings were transplanted to plastic cones $(9$ by $30 \mathrm{~cm}$ ) filled with sterilized quartz sand in the greenhouse with the average temperature of $25 \pm 3{ }^{\circ} \mathrm{C}$ and $20 \pm 2{ }^{\circ} \mathrm{C}$, and the relative humidity of $55 \pm 5 \%$ and $70 \pm 5 \%$ during day and night, respectively.

\section{Rhizobia inoculation and salt treatments}

After transplanting, all the seedlings were watered daily with a nutrient solution (Supplement Table S1) prepared according to Peel [49] with little modification. Sixty days after transplanting, seedlings with similar size were selected and randomly divided into three groups. The first group was inoculated with Rhizobium meliloti and watered with a N-free nutrient solution (Supplement Table S1), which resulted in development of active nodules (NA). The second group was also inoculated with the rhizobia but was watered with a complete nutrient solution, which led to the development of inactive nodules (NI) due to inhibition of nitrogen fixation by sufficient nitrogen in the nutrient solution. The third group was not inoculated and watered with a complete nutrient solution. These plants did not develop nodules (NN). Additional details of the treatments were described in other studies [39].

Plants were subjected to salt stress 30 days after inoculation. $\mathrm{NI}$ and $\mathrm{NN}$ plants were irrigated with a complete nutrient solution containing $150 \mathrm{mM} \mathrm{NaCl}$ and $\mathrm{NA}$ plants were irrigated with an $\mathrm{N}$-free nutrient solution containing $150 \mathrm{mM} \mathrm{NaCl}$. The controls were irrigated with respective nutrient solutions without $\mathrm{NaCl}$. The shoot tissues (leaves and stems) were harvested at 0, 5, 10 and $15 \mathrm{~d}$ post salt treatment. The tissues were immediately frozen in liquid nitrogen and stored at $-80^{\circ} \mathrm{C}$ until use.

\section{Determination of physiological changes in response to salt stress}

Relative water contents (RWC) of alfalfa leaves under salt stress were determined according to Chen et al. [50]. Dry matter was obtained after drying the shoot tissues in an oven at $80^{\circ} \mathrm{C}$ for $48 \mathrm{~h}$. The contents of proline and soluble sugars were determined spectrophotometrically by following the method of Bates et al.[51] and Dreywood [52] respectively. The activities of superoxide dismutase (SOD, EC 1.15.1.1) and catalase (CAT, EC 1.11.1.6) were measured by the nitroblue tetrazolium (NBT) method [53] and the method described by Raza et al.[54], respectively. Three plants from each treatment were used for measurement in each experiment. The experiment was repeated five times. Data were analyzed for mean difference by analysis of variance (ANOVA) in SPSS 20.0 with the least significant difference (LSD) test $(a=0.05)$. 


\section{Protein extraction and digestion}

The shoots of control ( $\mathrm{CK}$, treated with nutrient solutions without $\mathrm{NaCl}$ ) and salt-stressed ( $\mathrm{S}$, treated with nurient solutions containing $150 \mathrm{mM} \mathrm{NaCl}$ ) plants were harvested $10 \mathrm{~d}$ after stress treatment, when NA plants showed the greatest difference in performance compared to $\mathrm{NN}$ and $\mathrm{NI}$ plants based on the measurement of several physiological parameters. Tissues were ground into powder in liquid nitrogen and suspended in lysis buffer (8 M urea, 4\% CHAPS, $40 \mathrm{mM}$ Tris-HCl), with $1 \mathrm{mM}$ Phenylmethanesulfonyl fluoride (PMSF), $2 \mathrm{mM}$ Ethylenediaminetetraacetic acid (EDTA) and $10 \mathrm{mM}$ Dithiothreitol (DTT) (final concentration). The mixture was subjected to sonication for $5 \mathrm{~min}$, and centrifuged at $25,000 \times \mathrm{g}$ for 20 min. The supernatant was mixed with $10 \mathrm{mM}$ DTT and incubated at $56{ }^{\circ} \mathrm{C}$ for $1 \mathrm{~h}$, followed by a 45 -min incubation in a dark room after mixing with $55 \mathrm{mM}$ lodoacetamide (IAM) and a 2-h incubation at $-20{ }^{\circ} \mathrm{C}$ after mixing with five-volume of chilled acetone. The protein pellet was collected by centrifugation and stored at $-80^{\circ} \mathrm{C}$ for further use. For digestion, the protein pellet was resuspended in $0.5 \mathrm{M}$ triethylammonium bicarbonate (TEAB) solution, and the protein concentration was determined using bicinchonic acid assay (Sigma, USA). Equal aliquots $(100 \mu \mathrm{g})$ from each lysate were then digested with trypsin overnight at $37^{\circ} \mathrm{C}$ and lyophilized.

\section{ITRAQ labeling and strong cation exchange (SCX) chromatography}

The iTRAQ labeling of peptide samples derived from the shoots of alfalfa plants were performed using iTRAQ reagent 8-plex kit (Applied Biosystems, USA) according to the manufacturer's protocol with two biological replicates for each experimental group. All tagged samples were incubated at room temperature for $2 \mathrm{~h}$ and then were pooled together and lyophilized to powder form.

For SCX chromatography, the dried iTRAQ labeled and pooled samples were reconstituted with $4 \mathrm{~mL}$ buffer $\mathrm{A}\left(25 \mathrm{mM} \mathrm{NaH}_{2} \mathrm{PO}_{4}\right.$ in $25 \%$ acetonitrile (ACN), pH 2.7) and loaded onto a $4.6 \times 250 \mathrm{~mm}$ Ultremex SCX column (Phenomenex, USA) containing $5 \mu \mathrm{m}$ particles. The peptides were eluted at a flow rate of 1 $\mathrm{mL} / \mathrm{min}$ with a gradient of buffer $\mathrm{A}$ for $10 \mathrm{~min}, 5-35 \%$ buffer $\mathrm{B}\left(25 \mathrm{mM} \mathrm{NaH}_{2} \mathrm{PO}_{4}, 1 \mathrm{M} \mathrm{KCl}\right.$ in $25 \% \mathrm{ACN}, \mathrm{pH}$

2.7) for $11 \mathrm{~min}, 35-80 \%$ buffer $B$ for $1 \mathrm{~min}$. The system was then maintained in $80 \%$ buffer $B$ for 3 min before equilibrating with buffer $A$ for 10 min prior to the next injection. Elution was monitored by measuring absorbance at $214 \mathrm{~nm}$, and the fractions were collected every minute. The eluted peptides were pooled as 20 fractions, desalted by Strata XC18 column (Phenomenex, USA) and vacuum-dried.

\section{LC-ESI-MS/MS identification}

The mass spectroscopy analysis was performed using a TripleTOF ${ }^{\mathrm{TM}} 5600$ mass spectrometer (AB SCIEX, CA), fitted with a Nanospray III source and a pulled quartz tip as the emitter as described before [55]. Each 
fraction was resuspended in buffer $\mathrm{C}(2 \% \mathrm{ACN}, 0.1 \%$ formic acid $)$ and centrifuged at $20,000 \times \mathrm{g}$ for 10 min; $10 \mu \mathrm{L}$ supernatant was loaded on a Shimadzu LC-20AD nanoHPLC (Waters, USA) by the autosampler onto a $2 \mathrm{~cm}$ C18 trap column (inner diameter $200 \mu \mathrm{m}$ ), and the peptides were eluted onto a resolving $10 \mathrm{~cm}$ analytical $\mathrm{C} 18$ column (inner diameter $75 \mu \mathrm{m}$ ). Data were acquired using an ion spray voltage of $2.5 \mathrm{kV}$, curtain gas of $30 \mathrm{PSI}$, nebulizer gas of $15 \mathrm{PSI}$, and an interface heater temperature of $150{ }^{\circ} \mathrm{C}$. Duplicate injections for each of the fractions of each set were performed, and the results of the replicate injections were combined before database searching.

\section{Proteomic data analysis}

For protein identification, The raw MS/MS data was converted into MGF format by ProteoWizard tool msConvert, and the exported MGF files were searched using Mascot v2.3.02 (Matrix Science, UK) against with Medicago truncatula Mt4.0v1database

(ftp://ftp.jcvi.org/pub/data/m_truncatula/Mt4.0/Annotation/Mt4.0v1/), which contains 62,319 protein sequences. The search parameters were as follows: MS/MS fragment ion mass tolerance of $0.1 \mathrm{Da}$; enzyme specificity was set to trypsin; peptide tolerance was set at $0.05 \mathrm{Da}$; fixed modifications of carbamidomethylation at Cys and iTRAQ 8plex at Lys and the N-terminal amino group of peptides; variable modifications of oxidation at Met, iTRAQ 8plex at Tyr; monoisotopic mass was chosen. Proteins were further identified and quantified with an automated software IQuant [56]. The permutation test was used to estimate the statistical significance of the protein quantitative ratios. For each protein, a significance evaluation is corrected for multiple hypothesis testing by the Benjamini-Hochberg method.

The criteria of FDR $\leq 0.01$ was set for identification of peptides and proteins. At least one unique peptide was necessary for the identified protein. The significant expressed proteins were defined with a significant change $(\mathrm{P}<0.05$ and $>1.2$-fold or $<0.83$-fold change) in protein quantities between the compared samples in at least one repetition, with the other repetition displaying a similar trend. The stand division (SD) and $\mathrm{P}$ value for each protein was presented in the table (Supplementary Table S2 and Table S3) after the mean value of protein expression ratios of each compared groups. Clusters of Orthologous Groups of proteins (COG) were delineated by the source http://www.ncbi.nlm.nih.gov/COG/ [57]. Pathways enrichment analysis was done by MapMan software (version 3.5.1) with differentially expressed proteins [58].

\section{Results}

\section{Physiological responses to salt stress}

We examined leaf relative water content (RWC), superoxide dismutase (SOD) and catalase (CAT) activities, proline (PRO) and soluble sugar (SS) contents in alfalfa plants with active nodules (NA), inactive nodules (NI) and without nodules (NN) under different extents of salt stress (Fig. 1). RWC of alfalfa leaves decreased with the time of salt stress and was higher in inoculated plants (NA and $\mathrm{NI}$ ) compared to NN $11 \mathrm{~d}$ after salt stress treatment (Fig. 1A). The activities of SOD and CAT were found to be 
the highest in NA shoots under salt stress, and the difference was more noticeable on day 10 among NA, $\mathrm{NI}$, and NN plants (Fig. 1B and 1C). PRO and SS contents in NA and NI shoots were higher than those in NN after salt stress (Fig. 1D and 1E). A greater PRO content in NA shoots was observed on day 10 after stress compared to NI shoots. NA and NI plants also displayed higher SS contents on day 10 after salt stress compared to NN plants. Overall, NA plants show different physiological responses to salt stress treatment on day 10 compared to $\mathrm{NI}$ and NN plants, and the samples harvested on day 10 after salt stress were studied for difference in protein profiles among NA, NI and NN plants.

\section{Protein identification}

An iTRAQ-based quantitative proteomic approach was used in this study to investigate the molecular links between root nodules and alfalfa salt tolerance. With two biological replications, a total of 396,885 spectra were obtained, among which 33,896 spectra were matched to the reference genomes $\mathrm{M}$. truncatula protein database (ftp://ftp.jcvi.org/pub/data/m_truncatula/Mt4.0/Annotation/ Mt4.0v1/), representing 29,981 unique spectra. A total of 9,240 peptides were identified, representing 8,717 unique peptides which can be assembled into 3,274 proteins (Fig. 2A). Majority of the peptides (85\%) are 7- 17 amino acid residues long (Fig. S1). Over $50 \%$ of the 3,274 proteins identified contain at least two peptides (Fig. 2B).

The protein mass distribution was presented in Figure 2C. Proteins with $20-30 \mathrm{kDa}$ were the most abundant, followed by proteins with 30-40 and kDa 10-20 kDa. Our results showed that iTRAQ was able to identify proteins with a wide range of molecular mass. Forty one percent of the identified proteins had more than $10 \%$ of the peptide coverage, and $19 \%$ have more than $20 \%$ of the peptide coverage (Fig. $2 \mathrm{D}$ ).

\section{Repeatability analysis}

In this iTRAQ-based proteomics, the distribution of the coefficient of variation (CV) showed that our data had a relatively high repeatability with two biological replicates. The mean $\mathrm{CV}$ value is 0.17 (S_NA:CK_NA), 0.17 (S_Nl:CK_NI), and 0.19 (S_NN:CK_NN) for three comparative groups. When the level of variation was $<20 \%$, the proportion of the number of proteins was $0.70,0.69$, and 0.68 for S_NA:CK_NA, S_NI:CK_NI, and S_NN:CK_NN, respectively (Fig. 3).

\section{Overall differentially expressed proteins in alfalfa tissues}

A total of 3,274 non-redundant proteins in alfalfa shoots were identified (Supplementary Table S2). These proteins were used to determine the significant changes in abundance during salinity treatments. With a 1.2 -fold change cutoff and $p$-value less than $0.05,607$ proteins are differentially expressed in the shoots 
when comparing the salt stress condition with normal growth conditions in each treatment (Supplementary Table S3). Among the 607 proteins, 408 were up-regulated and 322 were down-regulated (Fig. 4). NN, NI, and NA shared 109 up-regulated and 61 down-regulated proteins. The numbers of proteins that are up-regulated specifically in $\mathrm{NN}, \mathrm{NI}$, and $\mathrm{NA}$ are 54,72 and 53 , respectively; and the numbers of proteins that are down-regulated specifically in $\mathrm{NN}, \mathrm{NI}$, and $\mathrm{NA}$ are 47,52 , and 55 . Pairwise comparison showed that $\mathrm{NI}$ and NA shared similar changes when each of $\mathrm{NI}$ or NA was compared to NN. $\mathrm{NI}$ and NA shared 79 up-regulated and 71 down-regulated proteins, while NN only shared 23 and 18 upregulated proteins with $\mathrm{NI}$ and $\mathrm{NA}$ and 25 and 11 down-regulated proteins with $\mathrm{NI}$ and NA, respectively.

\section{Functional classification of differentially expressed proteins}

Clusters of orthologous groups of proteins (COG) analysis was performed to predict the functions and phylogenetic annotations of differentially expressed proteins in NA, NI and NN tissues under salt stress (Fig. 5). NN, NI and NA all showed a higher up-regulation of proteins involved in "energy production and conversion (C)"; "amino acid transport and metabolism (E)", "carbohydrate transport and metabolism $(G)$ ", "translation, ribosomal structures and biogenesis $(\mathrm{J})$ "; "posttranslational modification, protein turnover, chaperones (0)" and "general function production only (R)". NA and NI generally showed similar but higher levels of changes when compared to $\mathrm{NN}$ in $\mathrm{C}$ and $\mathrm{E}$ functional categories. The most highly down-regulated functional categories are the same for NN, NI and NA, i.e. "translation, ribosomal structures and biogenesis $(\mathrm{J})$ "; "posttranslational modification, protein turnover, chaperones $(\mathrm{O})$ " and "general function production only (R)". Salt stress appeared to have little effect on "cell motility (N)". COG analysis for $\mathrm{NA}, \mathrm{NI}$ and $\mathrm{NN}$ plants showed that the three most down-regulated functional categories are among the six most up-regulation functional categories, and two of them, "translation, ribosomal structure, and biogenesis" and "posttranslational modification, protein turnover, chaperons", are involved in protein synthesis, modification or degradation, suggesting plants actively regulated protein quantity and activity during salt stress response, resulting in a large increase of proteins in "energy production and conversion", "amino acid transport and metabolism", and "carbohydrate transport and metabolism".

\section{Pathway analysis for differentially expressed proteins with MapMan}

To better understand how NA, NI and NN plants respond to salt stress differently, MapMan was used to compare the proteins involved in specific processes. As indicated in Figure 6, seven processes were upregulated in NA, NI and NN plants, and among them four processes are highly over-represented, and they are all related to photosynthesis. Three upregulated processes common to NA, NI and NN plants are under-represented and belong to "not assigned" categories (Fig. 6). Interestingly, the only down-regulated process common to NA, NI, and NN plant also belongs to "not assigned unknown" category. 
MapMan analysis revealed 5 processes were upregulated and 12 were downregulated only in NA under salinity based on the over-represented proteins (Fig. 6). Four of the up-regulated processes are related to cell wall modification. They include "cell wall degradation", "miscellaneous", "miscellaneous gluco-, galaco- and mannosidases" and "miscellaneous peroxidase". Among the 12 downregulated processes, 8 of them are related to protein metabolism, including two for protein degradation, four for protein synthesis and two for protein targeting/secreting. NA plants also showed downregulation of ethylene responsive proteins, receptor kinases and calcium signaling proteins.

Both NA and NI showed significant up-regulation of proteins involved in photosynthesis and respiration, including proteins in light reaction of PSI, in the Calvin cycle, such as fructose-bisphosphate adolase, in photorespiration, and in TCA cycle. NA and NI plants also showed a stronger up-regulation of proteins involved in biodegradation of xenobiotics and porin transport.

\section{Discussion}

Based on the physiological analysis, NA plants show higher RWC after 10 days of salt treatment compared to NN plants. Other biochemical and physiological assays indicated that NA plants showed significant changes as early as 5 days after salt treatment compared to NN plants, but the change reached the peak at 10 days after salt treatment in NA plants for most of the parameters analyzed. To understand the effect of nodules on salt tolerance of host plant, we analyzed the protein profiles among $\mathrm{NA}, \mathrm{NI}$ and NN plants on day 10 after salt stress. The analysis revealed processes and pathways that are involved in the response to salinity stress in NA, NI, and NN plants.

\section{Common pathway changes under salt stress}

As indicated in Figure 6, seven processes are upregulated in NA, NI and NN plants, and among them four processes are highly over-represented, and they are all related to photosynthesis. A large set of proteins involved in both light reaction and carbon fixation are up-regulated due to salt treatment. An increase in photosynthesis-related proteins under salt stress were also observed (Supplementary Table S4). Thus, the results suggested an enhancement of photosynthesis may be a common strategy to respond to salt stress, potentially to boost an energy source that is required for dealing with salinity stress. Notably, five chlorophyll binding a/b binding proteins (medtr2g008610.1, medtr2g081090.2, medtr4g015570.1, medtr5g097280.1, and medtr6g060175.1) were all up-regulated more than 2-fold only in NA plants, suggesting that the enhanced light energy harvesting may be needed to meet the additional energy needs for active nitrogen fixation. In the processes of light reaction, the light-harvesting chlorophyll a/b-binding proteins (LHC-CAB) absorb light through chlorophyll excitation and then transfer the absorbed energy to photo-chemical reaction centers [40]. These five proteins are members of chlorophyll binding complex in PSII, protect chlorophyll in cells, and play important roles in photosynthesis [41]. Three upregulated 
processes common to NA, NI and NN plants are underrepresented and belong to "not assigned" categories (Fig. 6). Interestingly, the only downregulated process common to $\mathrm{NA}, \mathrm{NI}$, and $\mathrm{NN}$ plant also belongs to "not assigned unknown" category.

\section{Unique pathway changes in NA plants}

We are more interested in the proteins that are uniquely impacted by active nitrogen fixation since we observed that active nitrogen fixation improves salt tolerance in alfalfa [39]. MapMan analysis revealed 5 processes were up-regulated and 12 were down-regulated only in NA under salinity based on the overrepresented proteins (Fig. 6).

Four of the upregulated processes are related to cell wall modification. They include "cell wall degradation" (Supplementary Table S5), "miscellaneous", "miscellaneous gluco-, galaco- and mannosidases" and "miscellaneous peroxidase" (Supplementary Table S6). Looking closely, several cell wall degradation enzymes are pectin lyases. Together with those galactosidases in the miscellaneous group, the data suggest that NA plants may actively decrease or modify pectin component in the wall. At the same time, some of the upregulated peroxidases may be involved in lignification or cross-linking of cell wall components that will strengthen cell walls [42]. It is well-known that salinity stress can severely disrupts synthesis and weakens cell wall structure [43]. These changes in NA plants may help maintain healthy status under salinity. Since peroxidases are also involved in removing ROS in plants, it is possible that some of the upregulated peroxidases are involved in reducing ROS molecules in NA plants. The physiological data (Fig. 1B and 1C) suggested that NA plants can indeed better deal with oxidative stress. We did not, however, identify upregulation of CAT and SOD proteins in our proteomic analysis, suggesting that the higher CAT and SOD activities in NA plants under salinity are due to posttranslational regulation. A cysteine-rich receptor-like kinase (Medtr2g088980.1) was only upregulated in NA plants, suggesting that nitrogen fixing plants may activate a unique signaling pathway that can enhance their tolerance to salinity stress (Supplementary Table S7).

Among the 12 down-regulated processes, 8 of them are related to protein metabolism, including two for protein degradation, four for protein synthesis and two for protein targeting/secreting (Fig. 6 and Supplementary Table S8). The data indicate that NA plants suppress protein synthesis and at the same time reduce protein degradation and secretion, implying NA plants may reduce the protein turnover rate to save energy from costly protein synthesis and efficiently use all the resource by reducing secretion. 
NA plants also showed down-regulation of ethylene responsive proteins (Supplementary Table S9). Ethylene is often considered a stress hormone, involved in suppression of growth [44].Thus a better stress tolerance and growth of NA plants under salt stress may be partially due to the reduced ethylene response. Several calcium-binding EF hand proteins (medtr3g115640.1, medtr5g011850.1, medtr5g088320.1, medtr5g099170.1, medtr8g107110.1) were down-regulated in NA, NI and/or NN plants (Supplementary Table S10). However, NA plants showed overall the strongest reduction in these proteins, and Meditr5g099170.1 was down-regulated only in NA plants. Calcium signaling plays an important role in plant response to various environmental stresses [45]. EF-hand proteins have been implicated in positive role in salinity tolerance in various plants, including Medicago [46]. The reason why multiple EFhand proteins were downregulated in alfalfa and NA plants is unknown.

\section{Common pathway changes in NA and NI plants}

Our previous studies demonstrated that NI plants also showed some improvement of salt tolerance compared to NN plants [39], prompting us to look at the pathways shared between NA and NI that may contribute to their salt tolerance. The MapMan analysis clearly showed many similar responses, mostly in up-regulated proteins, between NA and NI plants under salinity stress (Fig. 6). The high similarity was demonstrated in the proteins listed in Supplementary Tables S4-S13, in contrast with the changes in NN plants.

Both NA and NI showed significant up-regulation of proteins involved in photosynthesis and respiration, including proteins in light reaction of PSI, in the Calvin cycle, such as fructose-bisphosphate adolase, in photorespiration, and in TCA cycle (Supplementary Table S4 and Table S11). The data suggest that both NA and NI plants are actively managing energy production and consumption during stress response. Alternatively, these changes in carbohydrate metabolism may have something to do with a greater accumulation of osmoticans in NA and NA plants shown in Fig. 1 (1D and 1E) which may in turn contribute to a greater RWC in NA and NI plant after 10 days of salt stress in Fig. 1A. NA and NI plants both showed a stronger up-regulation of proteins involved in biotic stress response, including a chitinase (Medtr3g110320.2) and leucin-rich repeat protein (medtr3g009050.1) involved in defense response (Supplementary Table S12). It is possible that the biotic stress-related proteins are up-regulated under salinity, potentially a preventive strategy that NA and NI plants use to ensure a healthy status. Similar findings were also reported from M. de las Mercedes Dana [47] when plants were challenged with bacteria. Finally, both NA and NI plants both upregulated proteins in ion transport, including four voltagedependent anion selective channel (Supplementary Table S13). Salinity stress induced by $\mathrm{NaCl}$ results in both $\mathrm{Na}^{+}$and $\mathrm{Cl}^{-}$toxicity. In fact, excessive $\mathrm{Cl}^{-}$imposes stronger toxic effect on plants [48]. Thus, these anion channer may help maintain the anion homeostasis in alfalfa under salinity. 


\section{Conclusions}

In conclusion, we found that symbiotic interaction or nodulation of alfalfa altered the pattern of protein changes involved in multiple physiological processes during salinity stress response compared to the plants without nodulation. Our data suggested that NA plants may respond to salinity stress through regulating some unique signaling pathways, leading to a better tolerance to stress. The greater stress tolerance in NA plants may be associated with active cell wall remodeling and strengthening, enhanced activity of removing ROS molecules as well as suppressing protein synthesis and degradation. The data also suggest that NA plants, together with NI plants, enhance photosynthesis, carbon fixation and respiration, anion transport, and plant defense to pathogens. All these changes may allow NA plants to efficiently utilize all the energy and resources to maintain the healthy status, leading to better growth under salinity compared to $\mathrm{NI}$ and NN plants. Our study provides some strong supporting evidence for an improved salinity tolerance in NA and NI plants compared to NN plants [39]. While these observations are exciting, how NA plants regulate those specific processes is intriguing. Identification of signaling and regulatory mechanisms in symbiotic nitrogen-fixing alfalfa that enhance salinity tolerance in plants will be the focus of future research and may have a profound impact on agricultural production in saline soil.

\section{Abbreviations}

SNF: symbiotic nitrogen fixation; ROS: reactive oxygen species; iTRAQ: isobaric tags for relative and absolute quantification; RWC: relative water content; SOD: superoxide dismutase; CAT: catalase; PRO: proline; SS: soluble sugar; NA: alfalfa plants with active nodules; NI: alfalfa plants with inactive nodules; NN: alfalfa plants without nodules; COG: clusters of orthologous groups of proteins

\section{Declarations}

\section{Ethics approval and consent to participate}

Not applicable.

\section{Consent for publication}

Not applicable.

\section{Availability of data and materials}

Not applicable.

\section{Competing interests}

The authors declare that they have no competing interests.

\section{Funding}


This research was supported by the National Nature Science Foundation of China (31572456, 31772660), China Agriculture Research System (CARS-34), the USDA National Institute of Food and Agriculture Hatch Project 3AH390, South Dakota Agricultural Experiment Station, and the Department of Biology and Microbiology at South Dakota State University.

\section{Authors' contributions}

YW and YF analyzed and interpreted the proteomics data and drafted the manuscript; PZ conducted the experiment and drafted the manuscript; $\mathrm{TH}, \mathrm{PY}$ and $\mathrm{YW}$ designed the project. All authors read and approved the final manuscript.

\section{Acknowledgements}

Not applicable.

\section{References}

1. Singh S, Parniske M. Activation of calcium-and calmodulin-dependent protein kinase (CCaMK), the central regulator of plant root endosymbiosis. Curr Opin Plant Biol. 2012;15(4):444-53.

2. Sanchez-Canizares C, Palacios J. Construction of a marker system for the evaluation of competitiveness for legume nodulation in Rhizobium strains. J Microbiol Methods. 2013;92(3):246-9.

3. Boisson-Dernier A, Chabaud M, Garcia F, Bécard G, Rosenberg C, Barker DG. Agrobacterium rhizogenes-Transformed Roots of Medicago truncatula for the Study of Nitrogen-Fixing and Endomycorrhizal Symbiotic Associations. Mol Plant-Microbe Interact. 2001;14(6):695-700.

4. Franche $\mathrm{C}$, Lindström $\mathrm{K}$, Elmerich $\mathrm{C}$. Nitrogen-fixing bacteria associated with leguminous and nonleguminous plants. Plant Soil. 2009;321(1-2):35-59.

5. Dakora FD. Defining new roles for plant and rhizobial molecules in sole and mixed plant cultures involving symbiotic legumes. New Phytol. 2003;158(1):39-49.

6. Figueiredo M, Vilar J, Burity H. Alleviation of water stress effects in cowpea by Bradyrhizobium spp. inoculation. Plant Soil. 1999;207(1):67-75.

7. Yang PZ, Zhang P, Li B, Hu TM. Effect of nodules on dehydration response in alfalfa (Medicago sativa L.). Environ Exp Bot. 2013;86(2):29-34.

8. Yang J, Kloepper JW, Ryu C-M. Rhizosphere bacteria help plants tolerate abiotic stress. Trends Plant Sci. 2009;14(1):1-4.

9. Bray E. Plant responses to water deficit. Trends Plant Sci. 1997;2(2):48-54.

10. Zhu J-K. Salt and drought stress signal transduction in plants. Annu Rev Plant Biol. 2002;53(1):24773.

11. Munns R, Tester M. Mechanisms of salinity tolerance. Annu Rev Plant Biol. 2008;59:651-81.

12. Bertrand A, Dhont $C$, Bipfubusa M, Chalifour FP, Drouin P, Beauchamp CJ. Improving salt stress responses of the symbiosis in alfalfa using salt-tolerant cultivar and rhizobial strain. Appl Soil Ecol. 
2015;87:108-17.

13. Bruning B, van Logtestijn R, Broekman R, de Vos A, González AP, Rozema J. Growth and nitrogen fixation of legumes at increased salinity under field conditions: implications for the use of green manures in saline environments. AoB Plants. 2015;7.

14. Bouhmouch I, Souad-Mouhsine B, Brhada F, Aurag J. Influence of host cultivars and Rhizobium species on the growth and symbiotic performance of Phaseolus vulgaris under salt stress. J Plant Physiol. 2005;162(10):1103-13.

15. Lauter D, Munns D, Clarkin K. Salt response of chickpea as influenced by N supply. Agron J. $1981 ; 73(6): 961-6$.

16. Serraj R, Drevon JJ. Effects of salinity and nitrogen source on growth and nitrogen fixation in alfalfa. J Plant Nutr. 1998;21(9):1805-18.

17. Yousef A, Sprent J. Effects of $\mathrm{NaCl}$ on growth, nitrogen incorporation and chemical composition of inoculated and $\mathrm{NH}_{4} \mathrm{NO}_{3}$ fertilized Vicia faba (L.) plants. J Exp Bot. 1983;34(8):941-50.

18. Cordovilla MDP, Ligero F, Lluch $\mathrm{C}$. Effects of $\mathrm{NaCl}$ on growth and nitrogen fixation and assimilation of inoculated and $\mathrm{KNO}_{3}$ fertilized Vicia faba L. and Pisum sativum L. plants. Plant Sci. 1999;140(2):127-36.

19. Rao D, Giller K, Yeo A, Flowers T. The effects of salinity and sodicity upon nodulation and nitrogen fixation in chickpea (Cicer arietinum). Ann Bot. 2002;89(5):563-70.

20. Ertani A, Schiavon M, Muscolo A, Nardi S. Alfalfa plant-derived biostimulant stimulate short-term growth of salt stressed Zea mays L. plants. Plant Soil. 2013;364(1-2):145-58.

21. Becana M, Dalton DA, Moran JF, Iturbe-Ormaetxe I, Matamoros MA, C Rubio M. Reactive oxygen species and antioxidants in legume nodules. Physiol Plant. 2000;109(4):372-81.

22. Redondo FJ, de la Pena TC, Lucas MM, Pueyo JJ. Alfalfa nodules elicited by a flavodoxinoverexpressing Ensifer meliloti strain display nitrogen-fixing activity with enhanced tolerance to salinity stress. Planta. 2012;236(6):1687-700.

23. Chinnusamy V, Jagendorf A, Zhu J-K. Understanding and improving salt tolerance in plants. Crop Sci. 2005;45(2):437-48.

24. Hernandez J, Olmos E, Corpas F, Sevilla F, Del Rio L. Salt-induced oxidative stress in chloroplasts of pea plants. Plant Sci. 1995;105(2):151-67.

25. Bohnert HJ, Nelson DE, Jensen RG. Adaptations to environmental stresses. Plant Cell. 1995;7(7):1099.

26. Evelin H, Kapoor R, Giri B. Arbuscular mycorrhizal fungi in alleviation of salt stress: a review. Ann Bot. 2009;104(7):1263-80.

27. Szabados L, Savouré A. Proline: a multifunctional amino acid. Trends Plant Sci. 2010;15(2):89.

28. Rao KM, Raghavendra A, Reddy KJ. Physiology and molecular biology of stress tolerance in plants: Springer Science \& Business Media; 2006. 
29. Dong Z, Shi L, Wang Y, Chen L, Cai Z, Wang Y, Jin J, Li X. Identification and dynamic regulation of microRNAs involved in salt stress responses in functional soybean nodules by high-throughput sequencing. Int J Mol Sci. 2013;14(2):2717-38.

30. Yan S, Tang Z, Su W, Sun W. Proteomic analysis of salt stress-responsive proteins in rice root. Proteomics. 2005;5(1):235-44.

31. Chattopadhyay A, Subba P, Pandey A, Bhushan D, Kumar R, Datta A, Chakraborty S, Chakraborty N. Analysis of the grasspea proteome and identification of stress-responsive proteins upon exposure to high salinity, low temperature, and abscisic acid treatment. Phytochemistry. 2011;72(10):1293-307.

32. Swami AK, Alam SI, Sengupta N, Sarin R. Differential proteomic analysis of salt stress response in Sorghum bicolor leaves. Environ Exp Bot. 2011;71(2):321-8.

33. Wang MC, Peng ZY, Li CL, Li F, Liu C, Xia GM. Proteomic analysis on a high salt tolerance introgression strain of Triticum aestivum/Thinopyrum ponticum. Proteomics. 2008;8(7):1470-89.

34. Sobhanian H, Razavizadeh R, Nanjo Y, Ehsanpour AA, Jazii FR, Motamed N, Komatsu S. Proteome analysis of soybean leaves, hypocotyls and roots under salt stress. Proteome Sci. 2010;8(1):19.

35. Aghaei K, Ehsanpour A, Shah A, Komatsu S. Proteome analysis of soybean hypocotyl and root under salt stress. Amino Acids. 2009;36(1):91-8.

36. Kosová K, Vítámvás P, Prášil IT, Renaut J. Plant proteome changes under abiotic stress-contribution of proteomics studies to understanding plant stress response. J Proteomics. 2011;74(8):1301-22.

37. Munns R, Tester M. Mechanisms of salinity tolerance. Annual review of plant biology. 2008;59:65181.

38. Anower MR, Mott IW, Peel MD, Wu Y. Characterization of physiological responses of two alfalfa halfsib families with improved salt tolerance. Plant Physiol Biochem. 2013;71:103-11.

39. Wang Y, Zhang Z, Zhang P, Cao Y, Hu T, Yang P. Rhizobium symbiosis contribution to short-term salt stress tolerance in alfalfa (Medicago sativa L.). Plant Soil. 2016;402(1-2):247-61.

40. Green B, Durnford D. The chlorophyll-carotenoid proteins of oxygenic photosynthesis. Annu Rev Plant Biol. 1996;47(1):685-714.

41. Krol M, Spangfort MD, Huner NP, Oquist G, Gustafsson P, Jansson S. Chlorophyll a/b-binding proteins, pigment conversions, and early light-induced proteins in a chlorophyll b-less barley mutant. Plant Physiol. 1995;107(3):873-83.

42. Passardi F, Penel C, Dunand C. Performing the paradoxical: how plant peroxidases modify the cell wall. Trends Plant Sci. 2004;9(11):534-40.

43. Iraki NM, Bressan RA, Hasegawa P, Carpita NC. Alteration of the physical and chemical structure of the primary cell wall of growth-limited plant cells adapted to osmotic stress. Plant Physiol. 1989;91(1):39-47.

44. Burg SP. Ethylene in plant growth. Proc Natl Acad Sci. 1973;70(2):591-7.

45. Dodd AN, Kudla J, Sanders D. The language of calcium signaling. Annu Rev Plant Biol. 2010;61:593620. 
46. Wang T-Z, Zhang J-L, Tian Q-Y, Zhao M-G, Zhang W-H. A Medicago truncatula EF-Hand family gene, MtCaMP1, is involved in drought and salt stress tolerance. PLoS One. 2013;8(4):e58952.

47. de las Mercedes Dana M, Pintor-Toro JA, Cubero B. Transgenic tobacco plants overexpressing chitinases of fungal origin show enhanced resistance to biotic and abiotic stress agents. Plant Physiol. 2006;142(2):722-30.

48. Luo Q, Yu B, Liu Y. Differential sensitivity to chloride and sodium ions in seedlings of Glycine max and G. soja under $\mathrm{NaCl}$ stress. J Plant Physiol. 2005;162(9):1003-12.

49. Peel M, Waldron B, Jensen $K$, Chatterton N, Horton H, Dudley L. Screening for salinity tolerance in alfalfa: A repeatable method. Crop Sci. 2004;44(6):2049-53.

50. Chen D, Liang MX, DeWald D, Weimer B, Peel MD, Bugbee B, Michaelson J, Davis E, Wu Y. Identification of dehydration responsive genes from two non-nodulated alfalfa cultivars using Medicago truncatula microarrays. Acta Physiol Plant. 2008;30(2):183-99.

51. Bates LS, Waldren RP, Teare ID. Rapid determination of free proline for water-stress studies. Plant Soil. 1973;39(1):205-207.

52. Dreywood R. Qualitative test for carbohydrate material. Ind Eng Chem, Anal Ed. 1946;18(8):499.

53. Giannopolitis CN, Ries SK. Superoxide dismutases: I. Occurrence in higher plants. Plant Physiol. 1977;59(2):309-14.

54. Raza S, Athar H, Ashraf M, Hameed A. Glycinebetaine-induced modulation of antioxidant enzymes activities and ion accumulation in two wheat cultivars differing in salt tolerance. Environ Exp Bot. 2007;60(3):368-76.

55. Unwin RD, Griffiths JR, Whetton AD. Simultaneous analysis of relative protein expression levels across multiple samples using iTRAQ isobaric tags with 2D nano LC-MS/MS. Nat Protoc. 2010;5(9):1574-82.

56. Wen B, Zhou R, Feng Q, Wang Q, Wang J, Liu S. IQuant: an automated pipeline for quantitative proteomics based upon isobaric tags. Proteomics. 2014;14(20):2280-5.

57. Tatusov RL, Galperin MY, Natale DA, Koonin EV. The COG database: a tool for genome-scale analysis of protein functions and evolution. Nucleic Acids Res. 2000;28(1):33-6.

58. Thimm O, Bläsing O, Gibon Y, Nagel A, Meyer S, Krüger P, Selbig J, Müller LA, Rhee SY, Stitt M. MAPMAN: a user-driven tool to display genomics data sets onto diagrams of metabolic pathways and other biological processes. Plant J. 2004;37(6):914-39.

\section{Figures}

\section{Figure 1}


Effect of nodulation on physiological parameters of alfalfa under salt treatment. (A): relative water contents (RWC); (B): catalase (CAT) activity; (C): superoxide dismutase (SOD) activity; (D): proline (PRO) contents; (E): soluble sugar (SS) contents. NA: alfalfa plants with active nodules; NI: alfalfa plants with inactive nodules; NN: alfalfa plants without nodules. Data are shown as means $\pm S D(n=5)$. Different letters indicate significant difference $(\mathrm{p}<0.05)$ among $\mathrm{NA}, \mathrm{NI}$, and $\mathrm{NN}$ plants at the same day.
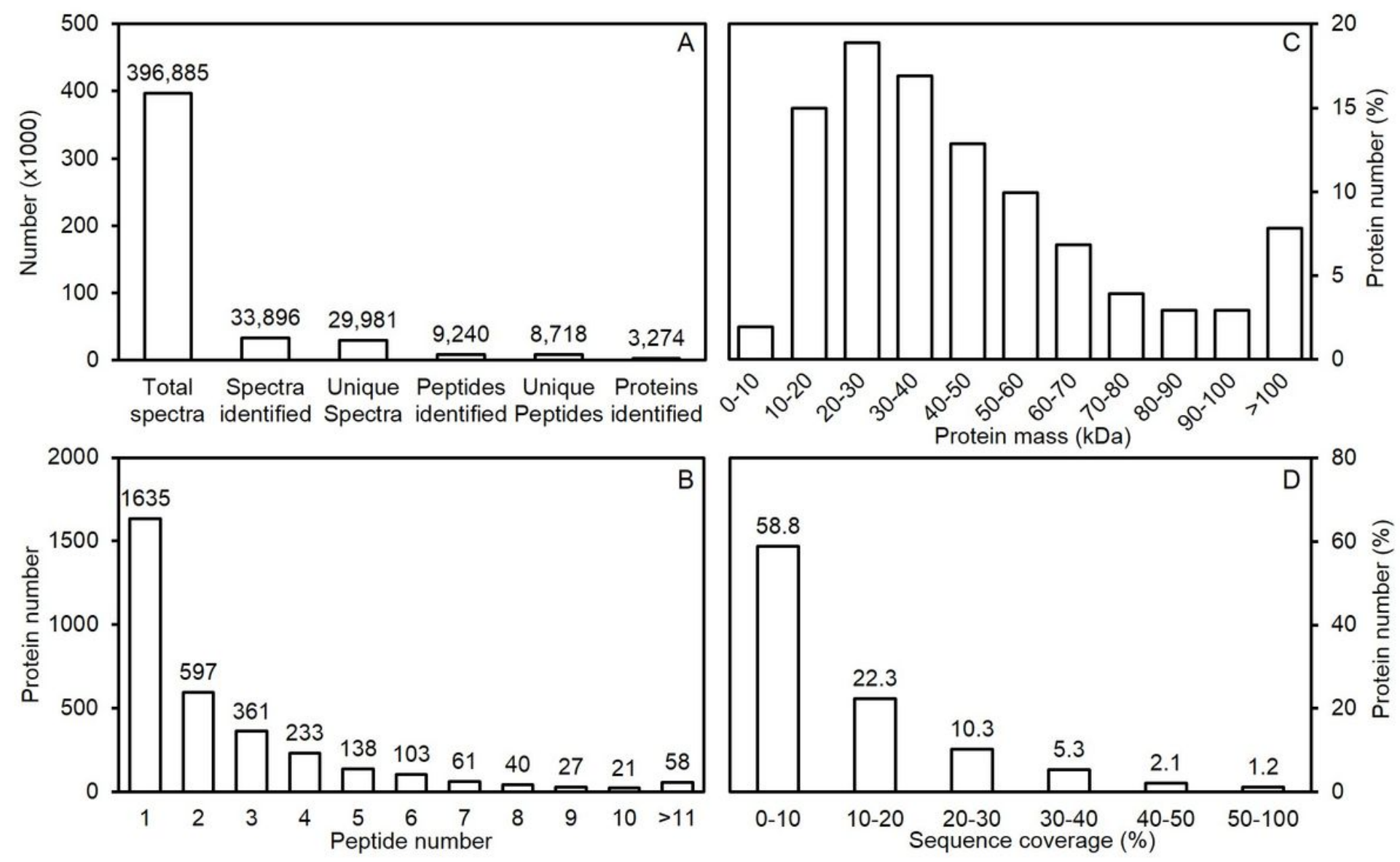

Figure 2

Protein identification. (A): spectra, peptides and proteins identified from iTRAQ proteomics by searching against database; (B): number of peptides that match to proteins using MASCOT; (C): protein mass distribution; (D): distribution of protein sequence coverage.

\section{Figure 3}

Repeatability analysis based on coefficient of variation (CV) of three comparison groups. (A) comparison between S_NA and CK_NA; (B) comparison between S_NI and CK_Nl; (C) comparison between S_NN and CK_NN. The left vertical axis indicates the percentage of the quantitative proteins at different levels of variation and the corresponding column, and the right vertical axis represents the cumulative percentage of proteins at different levels of variation and the corresponding line chart. S: plants under salt stress conditions; CK: plants under normal growth conditions; NA: alfalfa plants with active nodules; Nl: alfalfa plants with inactive nodules; NN: alfalfa plants without nodules. 


\section{Figure 4}

Venn diagrams of the differentially expressed proteins in the shoots when comparing the salt stress conditions with normal growth conditions in each treatment. The differentially expressed proteins were identified with a 1.2-fold cutoff and p-value less than 0.05. S: plants under salt stress conditions; CK: plants under normal growth conditions; NA: alfalfa plants with active nodules; NI: alfalfa plants with inactive nodules; NN: alfalfa plants without nodules. (A): the total 578 up-regulated proteins in NA, NI and NN groups; (B): the total 517 down-regulated proteins in NA, NI, and NN groups.

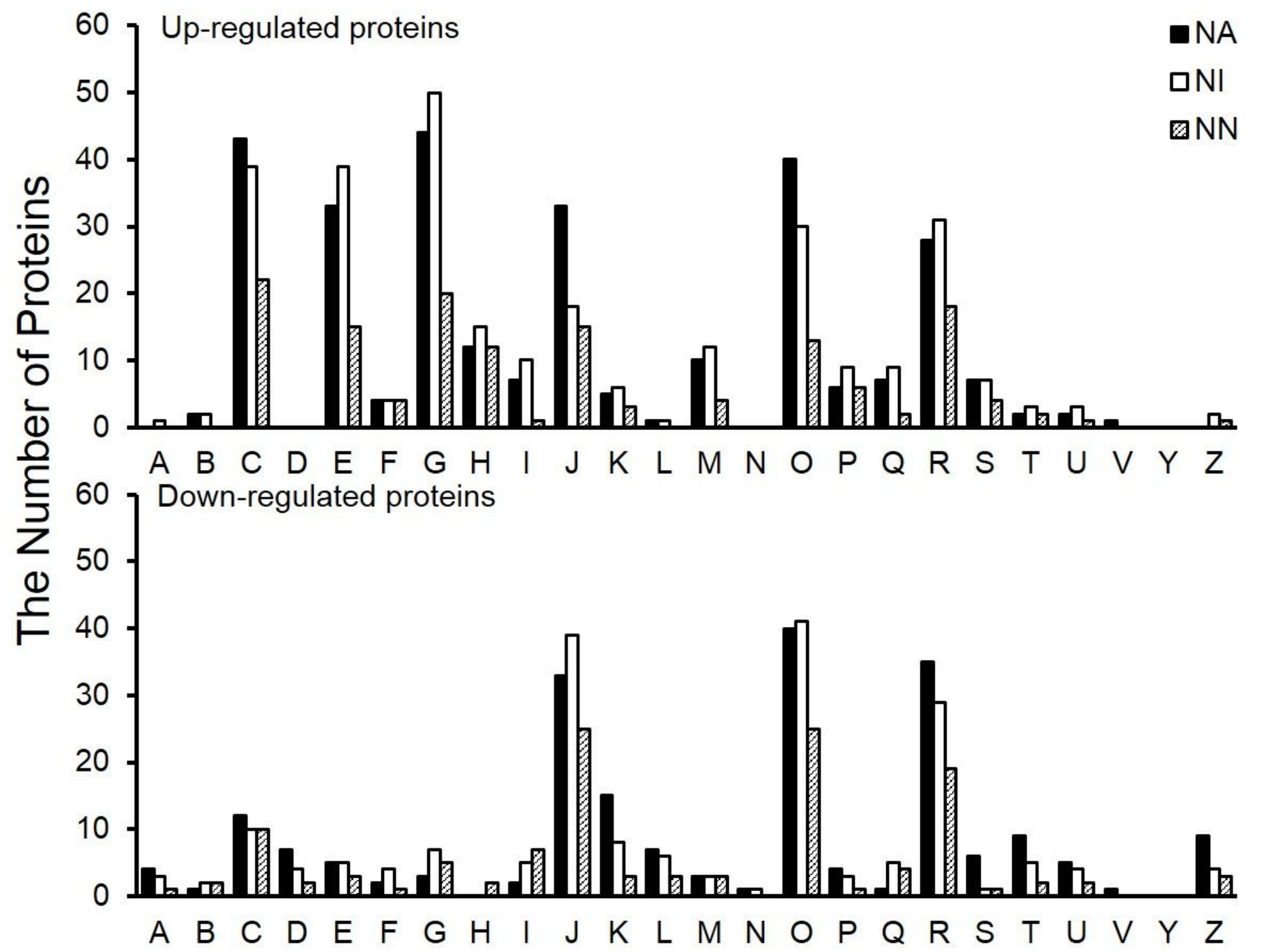

Figure 5

Clusters of orthologous groups (COG) of the differentially expressed proteins in alfalfa shoots when comparing the salt stress conditions with normal growth conditions in each treatment. The letters shown on X-axis mean different clusters. A: RNA processing and modification; B: Chromatin structure and 
dynamics; C: Energy production and conversion; D: Cell cycle control, cell division, chromosome partitioning; E: Amino acid transport and metabolism; F: Nucleotide transport and metabolism; G: Carbohydrate transport and metabolism; H: Coenzyme transport and metabolism; I: Lipid transport and metabolism; J: Translation, ribosomal structure and biogenesis; K: Transcription; L: Replication, recombination and repair; M: Cell wall/membrane/envelope biogenesis; $\mathrm{N}$ : Cell motility; O:

Posttranslational modification, protein turnover, chaperones; P: Inorganic ion transport and metabolism; Q: Secondary metabolites biosynthesis, transport and catabolism; R: General function prediction only; S: Function unknown; T: Signal transduction mechanisms; U: Intracellular trafficking, secretion, and vesicular transport; V: Defense mechanisms; Y: Nuclear structure; Z: Cytoskeleton.

\section{Figure 6}

Overrepresentation analyses of salt stress-responsive proteins defined by MapMan bins when comparing the salt stress conditions with normal growth conditions in each treatment. Fisher's exact test was used to identify functional categories enriched in up- or down-regulated proteins when protein abundances in $\mathrm{NA}, \mathrm{NI}$, and $\mathrm{NN}$ under salt stress conditions and normal conditions were compared. Blue, up- or downregulated proteins are significantly overrepresented; red, up- or down-regulated proteins are significantly underrepresented. Column 1, 2 and 3 represent the up-regulated pathways of NA, NI and NN under salt stress conditions compared with normal growth conditions, respectively. Column 4, 5 and 6 represent the down-regulated pathways of $\mathrm{NA}, \mathrm{NI}$ and $\mathrm{NN}$ under salt stress conditions compared with normal growth conditions, respectively. AGPs, arabinogalactan-proteins; LHC, light-harvesting complex; PS, photosynthesis; TCA, tricarboxylic acid cycle.

\section{Supplementary Files}

This is a list of supplementary files associated with this preprint. Click to download.

- S2totalprotein.xlsx

- S4S13MAPMANpathway.xIsx

- S3totalDEP.xlsx

- Fig.S1.jpg

- TableS1.docx 